\title{
Review Article \\ Optical Coherence Tomography in the Diagnosis and Monitoring of Retinal Diseases
}

\author{
Simona-Delia Tălu \\ Department of Surgical Specialties and Medical Imaging, Discipline of Ophthalmology, \\ "Iuliu Haţieganu" University of Medicine and Pharmacy, 8 V. Babeş Street, 400012 Cluj-Napoca, Romania
}

Correspondence should be addressed to Simona-Delia Ţălu; simonatalu@yahoo.com

Received 24 December 2012; Accepted 14 January 2013

Academic Editors: V. Positano, R. Tadeusiewicz, and J. Tang

Copyright (C) 2013 Simona-Delia Ţălu. This is an open access article distributed under the Creative Commons Attribution License, which permits unrestricted use, distribution, and reproduction in any medium, provided the original work is properly cited.

Optical coherence tomography (OCT) allows the visualization of the retinal microarchitecture as cross-sectional or tomographic volumetric data. The usefulness of OCT in the management of various retinal diseases is validated by the possibility to allow early diagnosis and to help in the decision-making process. OCT is applied by two main methods: time domain (TD-OCT) and spectral domain (SD-OCT). The advantages of SD-OCT over TD-OCT are significant improvement of the image axial resolution, decreased acquisition times, reduction of motion artifacts, increased area of retinal sampling, and the possibility to create topographic maps by the three-dimensional evaluation of tissues. OCT is the most precise method to measure the central macular thickness (which is the most important practical parameter) in vivo. It has been demonstrated that there are differences in the retinal thickness measurements between OCT models, explained by the higher axial and transverse resolutions of the newer devices. Further research has led to significant improvements in OCT technology represented by ultrahigh resolution OCT (UHR-OCT), swept source OCT (SS-OCT), enhanced depth imaging OCT (EDI-OCT), and adaptive optics. Technological progress in OCT imaging offered new perspectives for better understanding the retinal diseases, opening new avenues for the fundamental and clinical research. This is a review of the data in the literature concerning the evolution of OCT technology in the field of retinal imaging.

\section{Introduction}

The progress of the retinal imaging was determined by three major inventions: the direct ophthalmoscope, the fluorescein angiography, and the optical coherence tomography (OCT). The direct ophthalmoscope was invented by Hermann von Helmholtz in 1851 and gave access to the direct, in vivo visualization of the retina. In 1961 Novotny and Davis injected fluorescein dye intravenously in order to visualize the retinal circulation; thus, the technique named fluorescein angiography was born. In the 1990s OCT was invented which revolutionized the way we see the retina, by being able to visualize precisely the retinal layers-something done before only on pathology slides. Because of its simplicity, noninvasiveness, and richness of information, OCT became soon a crucial investigative tool in most of the retina practices [1].

Retinal diseases such as age-related macular degeneration (AMD), central serous chorioretinopathy, macular hole, vitreomacular interface syndrome, and diabetic maculopathy have taken advantage from the introduction of OCT in the clinical practice. The enormous advantages offered by OCT are expressed in terms of diagnosis, response to treatment, and monitoring of retinal diseases. The progress in the OCT techniques over the past decade is emphasized, from time domain-OCT (TD-OCT) to spectral domain-OCT (SDOCT) as well as future directions, with implications in the clinical practice.

\section{Theoretical Considerations on OCT Examination Techniques}

2.1. History. The OCT principle was born at Massachusetts Institute of Technology at the beginning of 1990s. Carl Zeiss (Jena, Germany) made available the first commercial version of OCT in 1996 [2]. The peripapillary area of the retina and the coronary artery were the first structures examined 
with OCT technology [3]. The first paper on time domain OCT (TD-OCT) was published by Huang and coworkers in 1991 [3] and in 1996 Hee et al. published the first data on the quantitative evaluation of the macular edema [4]. The continuous progress in OCT technology transformed this imaging method into a valuable examination tool for the retinal practice [5].

2.2. Optical Coherence Tomography versus Ultrasound. Ultrasounds deliver cross-sectional images of the eye with resolutions of $150 \mu \mathrm{m}$. If higher frequency waves are used, image resolutions of $20 \mu \mathrm{m}$ are obtained. The use of ultrasounds is limited to the anterior structures of the eye, because they are attenuated in the biological tissues [6]. OCT and ultrasounds are based on similar principles, with the difference that OCT uses light instead of sound. Because the light propagates nearly a million times faster than the sound, it allows the obtaining of much-higher-resolution images in the posterior pole of the eye: $10 \mu \mathrm{m}$ or less. OCT examination does not require the physical contact with the examined eye, making the examination more comfortable for the patient than ultrasounds [6].

2.3. Principle of OCT. Tomography consists in the reconstruction of cross-sectional images of an object by using its projections. OCT uses near-infrared light in order to deliver high-resolution cross-sectional images of the macula that are very similar to the histopathological specimens. OCT is based on low-coherence interferometry that uses light in order to measure the difference of reflectivity between the examined tissue and a reference beam. The detection of echoes resulting from this interference is measuring the light echoes versus depth $[7,8]$. However, the OCT images are not the direct depiction of the anatomical structures, but they represent the consequence of the optical properties of the tissues being scanned [9].

OCT is applied by two main methods: time-domain OCT (TD-OCT) and spectral-domain OCT (SD-OCT). TDOCT produces two-dimensional images of the examined tissue. In TD-OCT, the A-scans are gradually built up over time by moving a mirror in the reference arm of the interferometer and by collecting many A-scans OCT B-scans are generated. An A-scan represents a reflectivity profile in depth and a B-scan is a cross-section image, a lateral $\times$ depth map. In SD-OCT, information on depth is transformed from the frequency domain to the time domain, without using a moving reference mirror which allows the image to be acquired rapidly, about 60 times faster than with TDOCT. The SD-OCT units allow the improvement of the detection and monitoring of retinal diseases, because these ones have ultrahigh-speed scan rate, superior axial and lateral resolutions, cross-sectional (2D) scan, 3D raster scanning, and a higher imaging sensitivity than the traditional TDOCT units. Axial resolution is given by the light source: the bandwdith and the level of coherence that depends upon the central wavelength. The light from a superluminescent diode has long coherence that generates images with poor axial resolution, whereas with short-coherence light, interference

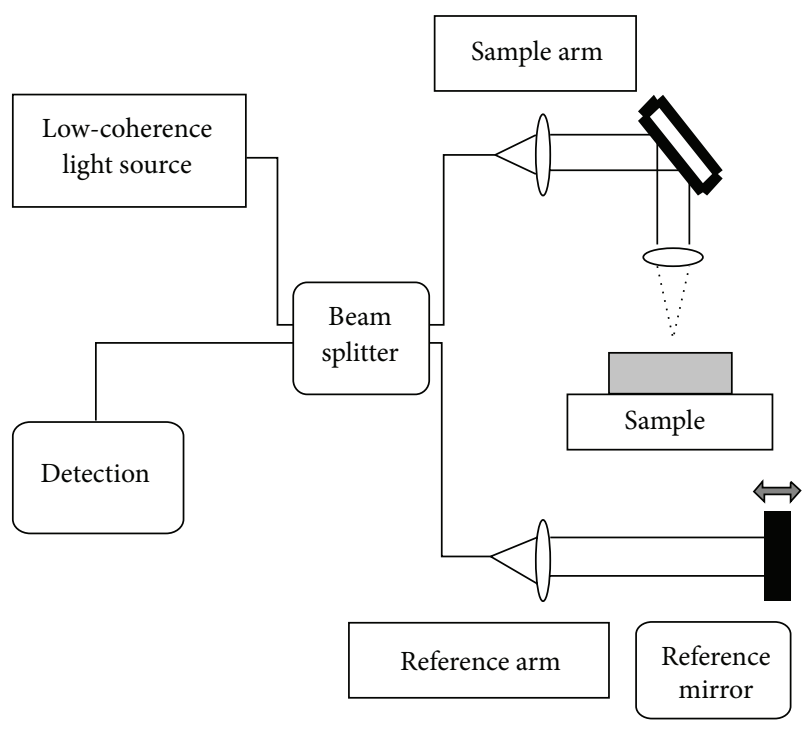

Figure 1: Principle of TD-OCT.

is possible over short distances and therefore high axial resolution is obtained [8]. The best structural resolution is obtained when the light is focused on the target tissue. The term "transverse resolution" refers to the size of the light spot that can be focused on the retina. The central light can be absorbed by the tissue of interest and this must be considered [8]. The SD-OCT software permits 3D reconstructions which can be further manipulated. All these advantages offered by SD-OCT create the possibility to demonstrate subtle pathology that cannot be objectivized with conventional 2D images [10].

2.4. TD-OCT (Conventional OCT). The principle at the origin of TD-OCT is low-coherence interferometry and is illustrated in Figure 1. The source of light is represented by a superluminescent diode that emits a radiation with the wavelength of $830-840 \mathrm{~nm}$. This emission is evenly divided into two beams by a beam splitter: a beam that enters the eye and is reflected back by the ocular media (named detection beam) and a beam that is reflected by a reference mirror (named reference beam). The two beams meet and the resulting interferences are intercepted by a light detector. The displacing of the reference mirror in various planes allows the visualization of different ocular structures, generating an A-scan. The B-scan of the retina, which is composed by a sequence of A-scans, is the result of the transverse scanning of the retina in a predefined axis (horizontal, vertical, or oblique). With this method cross-section images of the retina are obtained every 1.6 seconds (400 A-scans per second) [11]. The name of time domain which is given to this OCT method comes from the time which is required in order to get these sections. The quality of the image depends on two factors: the number of scans and the degree of light absorption by retinal and subretinal structures. The capability of TD-OCT system to produce high-quality images is defined by the value of signal-to-noise ratio (SNR) [10]. 
This technique generates a two-dimensional image and a cross-sectional map displayed in false colors in which each color is attributed a certain degree of reflectivity. White and red colors correspond to highly reflective tissues, whereas black and blue represent low-reflectivity structures and green is given an intermediate reflectivity. Fibrosis, haemorrhages, and infiltrates are hyperreflective tissues; therefore they will appear in red and white [6]. The retinal layers are displayed on the linear scans and the retinal thickness can be measured taking as references the vitreoretinal interface and the retinal pigmented epithelium, given their different reflectivity. By using 6 radial scans 30 degrees apart, a surface map is obtained, in which white and red represent high volume structures (e.g., macular edema) and black and blue correspond to thinned retinal areas [6].

The resolution of the OCT image is given by the coherence length of the light source. For the commercially available TD-OCT system, image axial resolution is in the range of $10-$ $15 \mu \mathrm{m}$. The penetration through transparent optical media is excellent but through a thick haemorrhage is less than $100 \mu \mathrm{m}$ [6].

The limits of TD-OCT are long acquisition times, limited image sampling (with the risk to overlook a small macular lesion), limited resolution generated by motion artifacts, and patient blinking [11].

2.5. SD-OCT (Spectral Domain-OCT). The SD-OCT devices use a central wavelength of $800-850 \mathrm{~nm}$, a stationary reference arm, a high-speed spectrometer that analyses simultaneously all the frequencies, and a charge-coupled device (CCD) line-scan camera. The Fourier mathematical equation (1807) is at the origin of SD-OCT development. The French mathematician Joseph Fourier described the decomposition of a periodic function into a sum of simple sinusoidal-based oscillating functions. The practical effect of this abstract statement is the possibility to measure simultaneously all echoes of light, in contrast to TD-OCT where the echoes of light are measured sequentially by moving a mirror in front of the reference beam. Subsequently, the image axial resolution in SD-OCT is much improved as compared to TD-OCT (3$7 \mu \mathrm{m}$ versus $10-15 \mu \mathrm{m}$ ). Therefore, the detection of individual retinal layers and lesions components became possible [12]. The practical impact of this improvement in resolution is the detection of small lesions that allow the early diagnosis and treatment. This is of particular importance in the neovascular form of age-related macular degeneration (AMD).

Given the possibility to get images simultaneously in various planes, the $3 \mathrm{D}$ reconstruction is possible with $\mathrm{SD}$ OCT, allowing the obtaining of hundreds of high-resolution images per second and the accurate measurement of the macula (total volume) in various conditions: edema, fluid, drusen, and choroidal new vessels (CNV). The acquisition speed increases to 25,000-52,000 A-scan/second and the amount of data that can be obtained during one session was improved significantly [13]. The reduction of the examination time considerably decreases the artifacts related to eye movements and poor fixation [14]. Another significant advantage of SD-OCT is represented by the increased retinal scan coverage. The SD-OCT images have proven to be clearer and with higher quality as compared to the ones obtained by the successive TD-OCT systems (OCT1, OCT3, Stratus). The SD-OCT systems are continuously improving, by adding complementary functions: fundus photography, angiography, and microperimetry. The ultrahigh-resolution images obtained by SD-OCT allow a better differentiation between the retinal and subretinal layers [2].

Several SD-OCT instruments are available at the current moment: Cirrus HD-OCT (Carl Zeiss Meditec), RTVue-Fourier Domain OCT (Optovue), Copernicus OCT (Reichert/Optopol Technology), Spectral OCT/SLO (Opko/ Oti), Spectralis HRA+OCT (Heidelberg Engineering), Topcon 3D OCT-1000 (Topcon), and RS-3000 Retiscan (Nidek). All the above-mentioned instruments provide highquality images and offer the possibility of tridimensional reconstruction of the macula [15].

In Figures 2(a) and 2(b), the normal aspect of the macula is illustrated. Figure 2 (a) represents the $3 \mathrm{D}$ visualization of a macular cube of $512 \times 128$ and Figure $2(\mathrm{~b})$ shows the macular thickness. The images were obtained with the Cirrus OCT which is an SD-OCT device. In both images the foveal pit is evident, as well as the normal, regular arrangement of the retinal layers, resembling a histological section of the macula. The highly reflective tissues are given the red color (retinal pigment epithelial band, internal limiting membrane), whereas the medium reflective layers of the neurosensory retina are represented in green. The macular thickness appears normal: $269 \mu \mathrm{m}$.

\section{Applications of OCT in Ophthalmology}

OCT is the most precise method to measure retinal thickness in vivo. However, there is not a direct correlation between OCT measurements and the histopathologic ones, because OCT signals are directly determined by the optical properties of tissues. Therefore, structures that stain strongly on the histopathological specimens do not necessarily appear as intense OCT signals. Another observation derives from the differences that were identified between OCT models, possibly explained by the higher axial and transverse resolutions of the newer devices. For instance, a comparison was made between Cirrus and Stratus OCT devices in measuring the macular thickness [16]. Firstly, the definition of the retinal boundaries used by the automated segmentation algorithms differs between devices [16]. The Stratus OCT program measures between the nerve fiber layer and the inner limit of the RPE complex, though it has been reported that Stratus OCT has two outer reference lines: one at the junction between the inner/outer segment of the photoreceptor cells and the other at the inner boundary of the RPE. The Cirrus OCT program measures the retinal thickness between the nerve fiber layer and the external band of the RPE. Subsequently, the Cirrus outer reference band is deeper than the first-mentioned Stratus external band and is closer to the second-mentioned one. The correlation of thickness measurements between the two devices is modest, as the Cirrus OCT provides greater measurement depth. Therefore, 


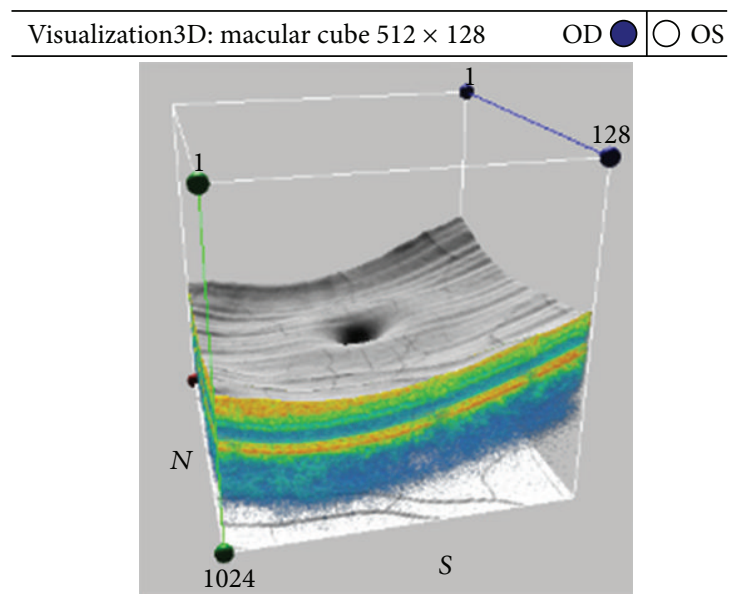

(a)

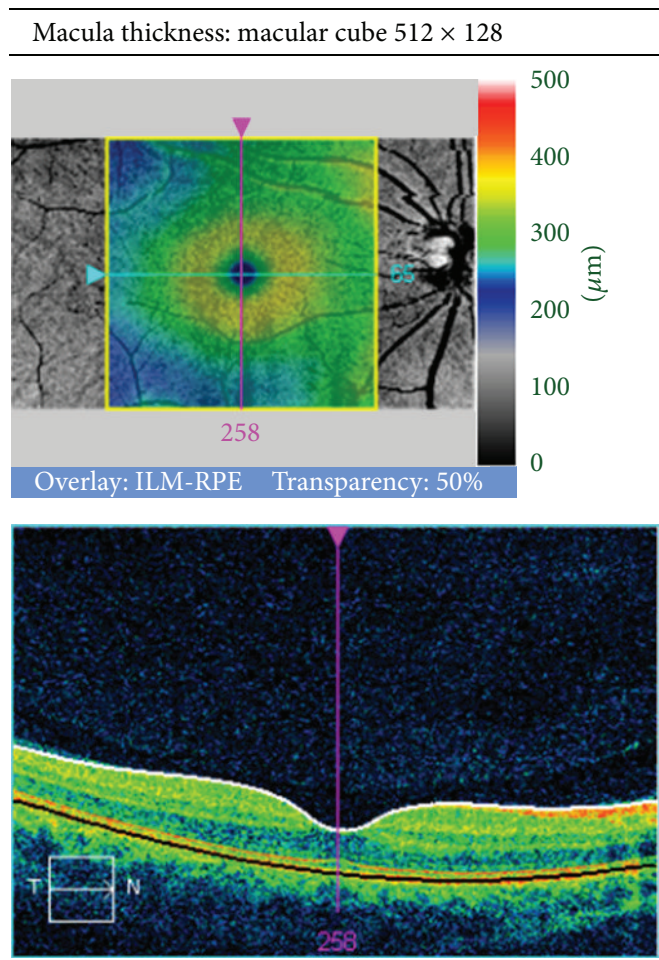

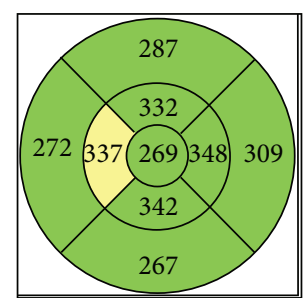

ILM-RPE thickness $(\mu \mathrm{m})$

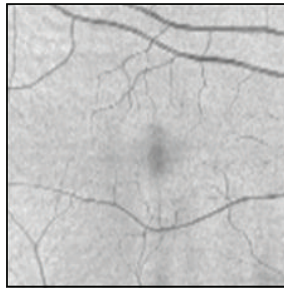

Fovea: 258,65

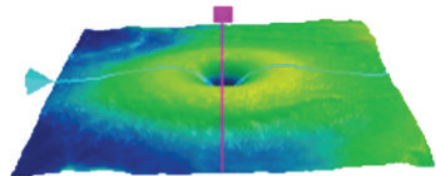

ILM-RPE

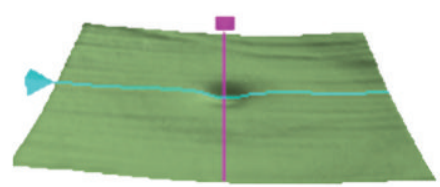

ILM

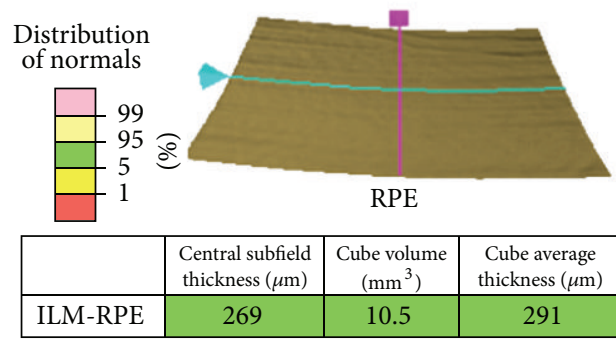

(b)

FIgURE 2: (a) and (b): Normal OCT images of the macula. 
TABLE 1: OCT advantages in the evaluation of macular edema.

(i) OCT is more reliable than fundus biomicroscopy in the evaluation of the macular edema.

(ii) OCT is more reliable than fluorescein angiography in diagnosing the presence of fluid in the retinal and subretinal space, as on fluorescein angiography the leakage in the inner retina can mask the fluid in the outer retina.

in the clinical practice the patients participating in clinical trials may not interchange between Stratus and Cirrus OCT systems and the patients transiting from one practice to another should have the tests done with the same OCT model. On the other hand, the manufacturer should upgrade the Stratus OCT software. Secondly and more importantly, the retinal thickness measured with TD-OCT is calculated based on 6 radial line scans, whereas with SD-OCT the 3D scan data are the result of 128-200 scans over the same area [16]. Thus, TD-OCT evaluates a small macular area as compared to SDOCT that images almost the entire macular region.

The advantages of OCT over fundus biomicroscopy and fluorescein angiography in evaluating the macular edema are summarized in Table 1.

3.1. OCT in Age-Related Macular Degeneration (AMD). $\mathrm{AMD}$ is the ocular disease that benefited the most from the development of the OCT technology. OCT demonstrated its usefulness in better understanding the pathogenesis of this disease, establishing the prognostic criteria and evaluating the response to the treatment with anti-vascular endothelial growth factors (VEGF) in the wet form of the disease.

3.1.1. OCT Imaging of $C N V$. In neovascular AMD, the retina is invaded by new vessels originating in the choroidal vessels. In the recent years, OCT proved its usefulness in defining the features of occult CNV. The occult choroidalneo vascularization $(\mathrm{CNV})$ is revealed by the constant presence of the elevation or detachment of the RPE band. Frequently, the occult $\mathrm{CNV}$ is suggested by various alteration of the RPE: irregularities, fragmentation, thickening, thinning. The RPE elevation is poorly delineated and its extent is variable according to the stage of the lesion. In occult CNV, the RPE elevation can be associated with other OCT signs: modifications of the RPE band (hyperreflectivity, fragmentations, thickening, thinning), subretinal and intraretinal accumulation of fluid, vitreomacular traction syndrome [17]. The subretinal fluid appears as diffuse infiltration or as the constitution of cystic spaces in the macular area.

The classic CNV is translated on OCT as hyperreflective zones adjacent to or away from the RPE. They must be differentiated from other hyperreflective structures: fibrous tissue, exudate, pigment, pseudovitelliform material.

Other signs of prognostic value can be visualized at the level of the outer retinal layers (outer nuclear layer and external limiting membrane): hyperreflective spots and areas of densification. They prove the progression of the disease [18].
3.1.2. OCT Imaging of Pigment Epithelial Detachments (PEDs). Of all the ophthalmological diseases, AMD is the condition in which PEDs are identified the most frequently. The PEDs usually indicate the presence of $\mathrm{CNV}$ and are associated with poorer visual outcome. Therefore, their identification carries a prognostic value $[19,20]$.

Using a TD-OCT device, which was not able to visualize within the PED, Coscas was the first to observe that the presence of a layer of tissue behind the RPE in PEDs is associated with occult CNV. With the help of the new devices the description of the PEDs content became possible: the dark regions within them are not attributable to the low sensitivity of the imaging technique, but to the hyporeflectivity of the region itself [19]. Cirrus SD-OCT system can be used in order to measure the area and volume of PEDs with the help of a highly reproducible algorithm which is similar to the one reported for the measurement of drusen [20].

3.1.3. OCT Imaging of Vitreomacular Adhesions. Several authors showed that vitreous may play a role in AMD pathogenesis and/or progression [21]. It was proved that the prevalence of posterior vitreous detachment (PVD) was significantly lower in patients with AMD as compared with healthy subjects [22]. In patients with partial PVD, the region of vitreomacular adhesion corresponded to the area of subretinal neovascularization [23]. Also, the incidence of persistent vitreomacular adhesion was significantly higher in eyes with wet AMD as compared to those with dry AMD and controls. If future prospective studies will confirm these findings, it seems logical to use OCT in order to assess the vitreomacular relationship in AMD patients. This is important, as the OCT findings could guide the prevention of wet AMD, by indicating the vitreomacular separation, either by vitrectomy or by pharmacologic vitreolysis [21-23].

3.1.4. Choroidal Thickness Measurements. Choroid is a crucial structure for AMD, as choroidal circulatory abnormalities seem to be the most significant factor involved in the development of this disease. The use of the newest OCT software enables the measurement of choroidal thickness, but only few studies evaluated it in AMD. So far, it has been proved that the choroidal thickness is variable among patients with AMD. An overall conclusion is that the patients with wet AMD displayed thinner choroids than the ones with dry AMD. These findings have also pathogenetic implications: the continuing thinning of the choroid might be a factor indicating the risk of progression towards wet AMD. The thinner than normal choroid induces ischemia at the level of RPE, stimulating the production of vascular endothelial growth factor (VEGF) with the subsequent proliferation of new vessels [24].

3.1.5. OCT in Dry AMD. In dry AMD there is atrophy of the choroid with subsequent degeneration of RPE and involution of the external retinal layers, including the photoreceptors. However, even if the RPE becomes atrophic, it preserves the barrier function, keeping the macular region dry [25]. The current OCT systems allow the identification of drusen. 


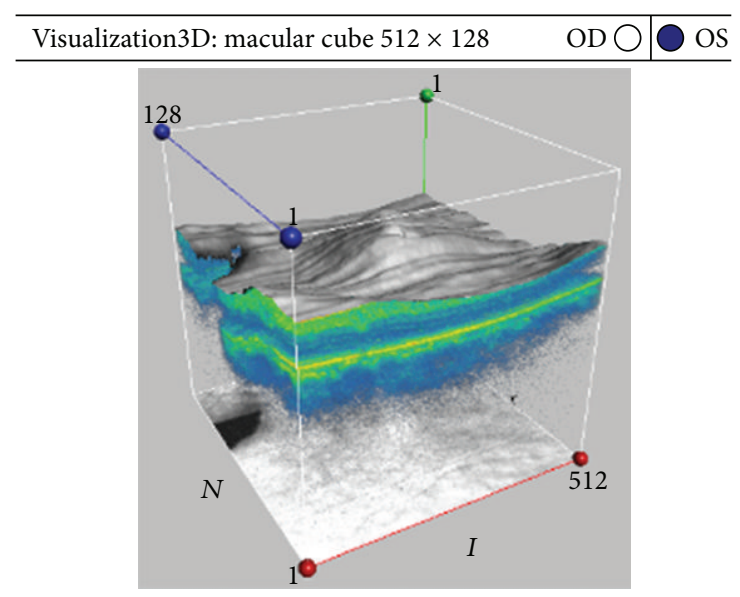

(a)

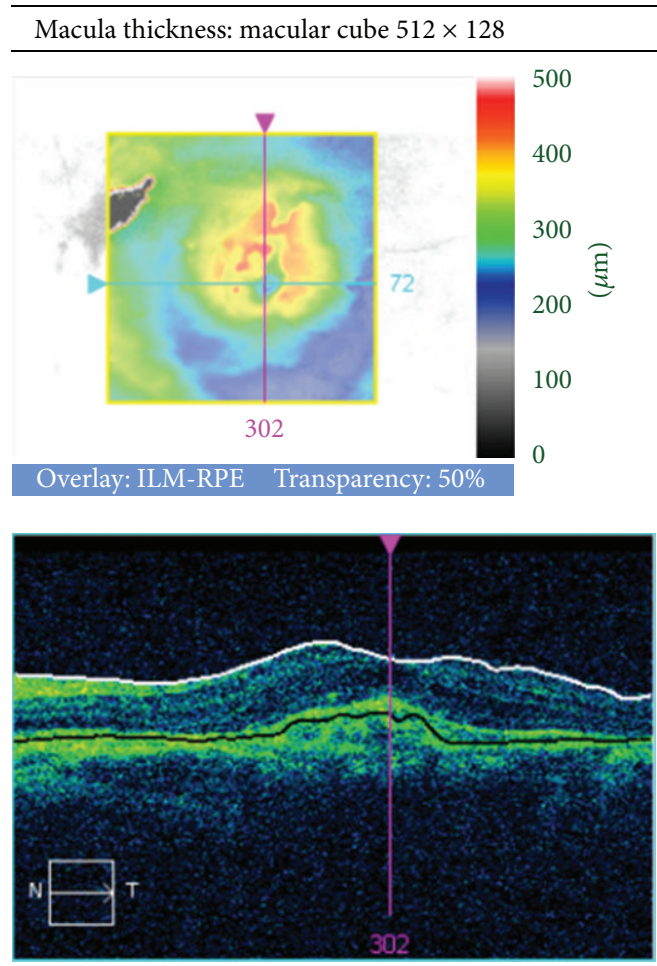

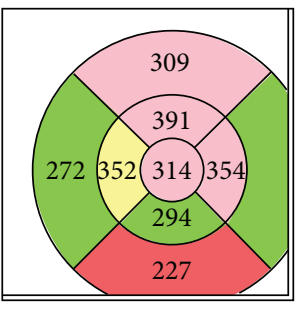

ILM-RPE thickness $(\mu \mathrm{m})$ OD $\bigcirc \longdiv { O \text { OS } }$

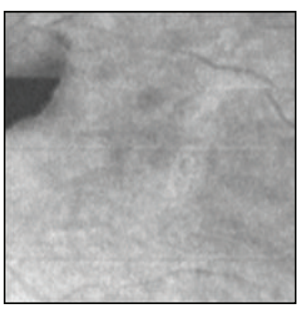

Fovea: 302,72

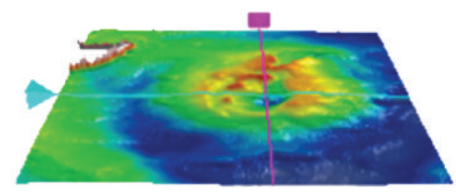

ILM-RPE

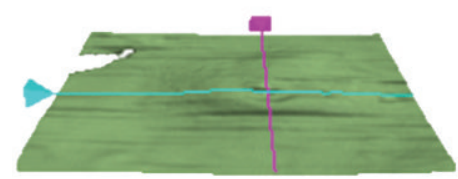

ILM
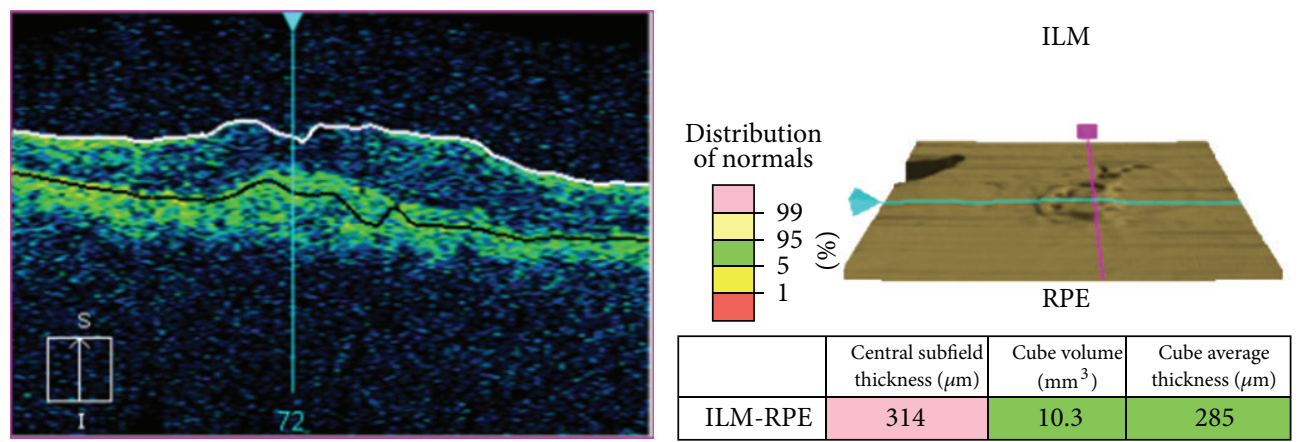

(b)

Figure 3: (a) and (b): Macular edema in wet AMD. 


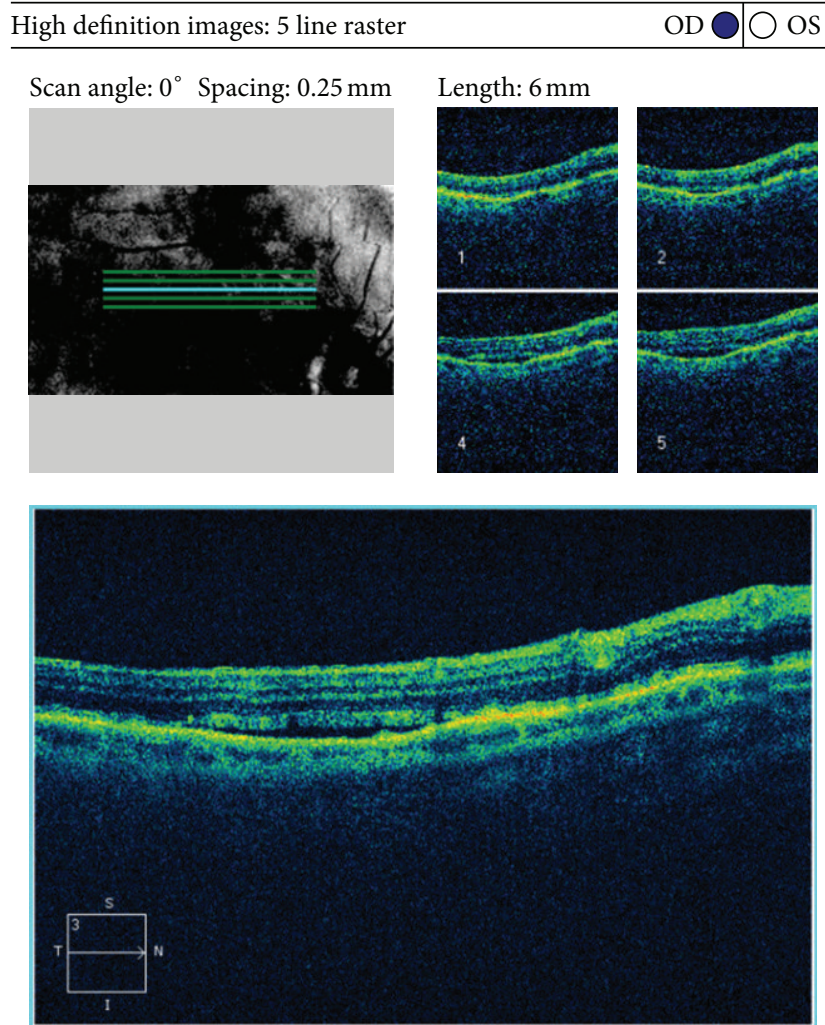

(a)
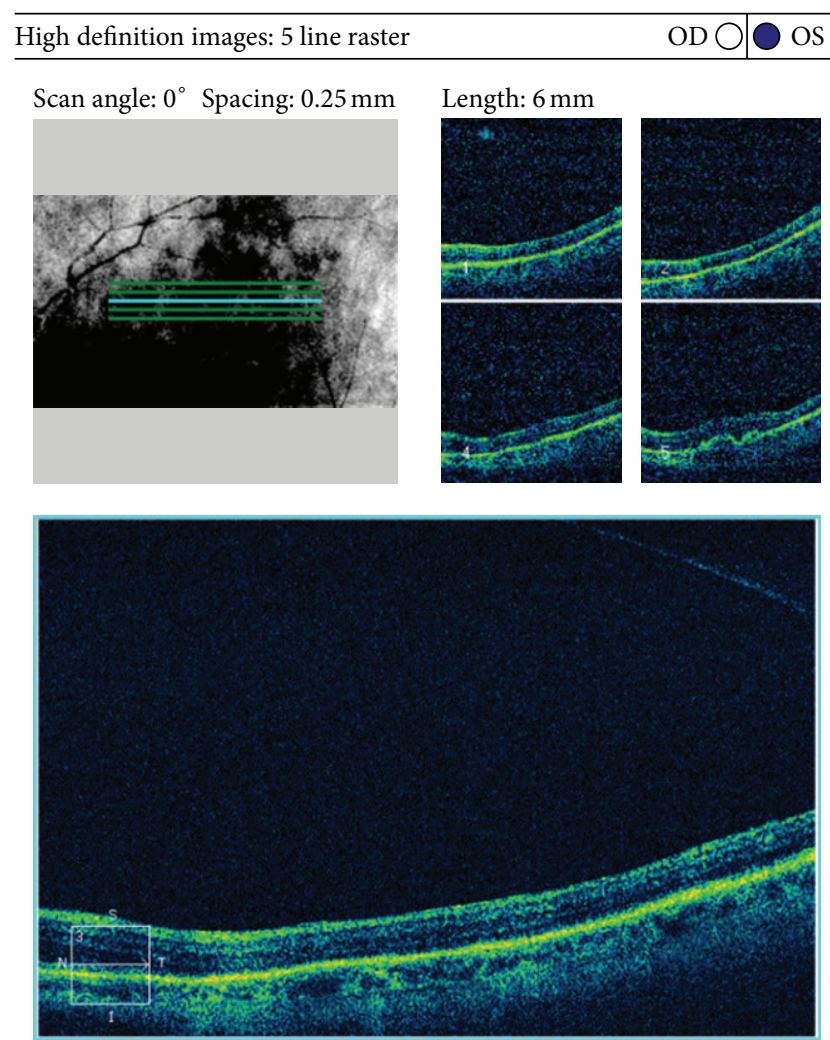

(b)

Figure 4: (a) and (b): Bilateral AMD.

The small- and intermediary-size ones appear as discrete elevations of the RPE with variable reflectivity, according to the underlying material. In the large drusen (or so-called drusenoid PEDs) the RPE displays greater, often domeshaped elevations separated from the Bruch membrane by a hypo- or medium-reflective material. On OCT images drusen are often accompanied by modifications in the neurosensory retina, translated by the thinning of the outer nuclear layer and disruption at the level of the external limiting membrane and of the inner segment (IS)-outer segment (OS) junctions [26]. Geographic atrophy (GA) appears on OCT as sharply demarcated areas of choroidal hyperreflectivity due to the loss of RPE. If retinal atrophy is associated, the following modifications are identified: thinning or loss of the outer nuclear layer, absence of the external limiting membrane and of the IS-OS junctions [27]. The evaluation of GA in terms of size, location, and rate of progression is crucial in assessing the visual prognosis of the patients. OCT reveals dynamic changes in the junctional zones of GA: pigment migration, variation in drusen height. In conclusion, OCT examination in dry AMD is important in two main directions: it brings new insights in the pathogenesis of the disease and it allows the prediction of the visual outcome [26].

Figure 3(b) shows the elevation of the RPE band which is irregular by a hyperreflective structure corresponding to a CNV membrane. The macular thickness is increased to
$314 \mu \mathrm{m}$. The macular edema is obvious also on the macular cube in Figure 3(a).

Figure 4(a) represents a wet form of AMD, diagnosed on the ground of RPE band irregularities associated with optically empty spaces corresponding to the accumulation of fluid under the neurosensory retina (above the RPE band). In Figure 4(b) the exudation is not yet identified, but there are some elevations of the RPE band representing drusen (Table 2).

3.2. OCT in Diabetic Macular Edema (DME). DME is the most common cause of vision loss in the diabetic patients. Before the OCT era, the DME was defined according to clinical criteria. OCT is more sensitive than fundus biomicroscopy in identifying DME, imposing the definition of a new entity: subclinical foveal edema, with possible therapeutical implications. Three different patterns have been described among patients with DME, but all of them have a common feature: the macular thickening [6]. The three patterns are: sponge-like swelling of the macula, cystoid macular edema, and serous macular detachment. The identification of these three subgroups is important in predicting the evolution of edema: the sponge-like type has a better outcome than the other two subtypes. Another major breakthrough of OCT in DME is the capability to identify the relationship between the posterior hyaloid and the macula. The presence of vitreomacular adhesion in DME patients is a key element in 
TABLE 2: OCT in AMD.
(i) OCT is able to describe accurately the CNV in AMD and its relation to the RPE and the neurosensory retina.
(ii) OCT reveals CNVs that on fluorescein angiography are masked by pooling of dye or by thin haemorrhages.
(iii) OCT revealed that cystoid macular edema is strongly correlated with the classic CNV.
(iv) OCT is crucial for the evaluation of the response to anti-VEGF intravitreal injections and the indication of retreatment.
(v) OCT brought significant contributions in understanding the pathogenesis and predicting the visual outcome in patients with GA.

TABLE 3: The role of OCT in DME.

(i) The major contribution of OCT in DME is the identification of hyaloidal-macular traction, establishing the indication of pars plana vitrectomy.

(ii) OCT allows the detection of subclinical macular edema, with possible therapeutical implications.

(iii) In DME there is a correlation between macular thickness measured by OCT and visual acuity.

TABLE 4: OCT in CRVO and BRVO.

(i) There is no correlation between macular thickness measured by OCT and visual acuity in CRVO and BRVO.

(ii) In retinal vein occlusions the visual acuity drops early and deeply and the residual vision depends rather on the state of the retinal circulation than on the macular thickness.

(iii) In CRVO and BRVO, OCT evaluates the evolution of the macular thickness after surgical intervention and identifies the subclinical retinal detachment.

the favor of pars plana vitrectomy for treating this condition [6]. The role of OCT in DME is presented in Table 3.

Figures 5(a) and 5(b) illustrate a cystoid macular edema in a diabetic patient, determined by vitreomacular traction. The macular thickness is considerably increased $(575 \mu \mathrm{m})$ and correlated to the poor visual acuity. Posterior vitrectomy with internal limiting membrane peeling was not followed by anatomical and functional success, because of the irreversible damage of the macular structure determined by the prolonged vitreal traction.

3.3. OCT in Central and Branch Retinal Vein Occlusion. Most of the signs in central and retinal vein occlusion (CRVO, BRVO), such as macular edema, retinal haemorrhages, and cotton wool spots, are well displayed on the OCT images. The accumulation of fluid in the outer retina appears as a hyporeflective area on OCT. If the roof of a cyst breaks, a macular hole may form, interrupting the reflectivity of the inner retina with variable depth [6]. A serous subretinal detachment may occur, appearing on OCT as a regular hyporeflective band located above the hyperreflective band representing the retinal pigmented epithelium. It is very important to differentiate this hyporeflective band from the shadow effect given by a retinal haemorrhage which appears as reflective as the retina. The aspect of the cotton wool spots is that of elevated hyperreflective structures that attenuate the underlying reflectivity. The progression of the edema is evaluated by the measurement of the retinal thickness. However, unlike in DME, there is no correlation between the macular thickness and the visual acuity. This lack of correlation seems to be attributed to the fact that in CRVO there is a deep drop in visual acuity from the beginning and the residual vision is determined by the condition of the retinal circulation, not by the macular thickness. The OCT is valuable in CRVO and BRVO by its capability to determine the macular thickness before and after surgical intervention. It also identifies subclinical retinal detachment which explains the difficulty of performing laser photocoagulation in some of these cases [6]. The particularities of OCT in CRVO and BRVO are summarized in Table 4.

3.4. OCT in Uveitis. OCT is as sensitive as fluorescein angiography in identifying macular edema in uveitis and has the advantage of better depicting its distribution within the macular area and diagnosing the subretinal fluid accumulation. When compared to fundus biomicroscopy, OCT is more sensitive in detecting active lesions and neovascular membranes, with the practical impact of earlier treatment [6]. There is a poor correlation between macular thickness and visual acuity in uveitic macular edema, attributable to the irreversible damage of the retina caused by chronic cystoid macular edema. The usefulness of OCT in uveitic macular edema is of particular importance in patients with posterior synechiae in which fundus biomicroscopy and fluorescein angiography are difficult to perform [6]. The features of OCT imaging in patients with uveitis are illustrated in Table 5.

3.5. OCT in Vitreoretinal Interface Syndrome. Vitreoretinal adhesions and traction are involved in the pathogeny of various diseases: vitreomacular traction syndrome, macular hole, epiretinal membranes, and cystoid macular edema [6]. Vitreoretinal traction induces macular thickening and fluid accumulation within the macula. OCT brought a significant contribution in describing the patterns in vitreoretinal interface syndrome. Two situations were identified: focal adhesion to the fovea or parafoveal region associated with 
TABLE 5: OCT in uveitic macular edema.

(i) In uveitic macular edema there is a poor correlation between macular thickness measured by OCT and visual acuity.

(ii) This poor correlation is explained by the irreversible damage of the retina caused by chronic cystoid macular edema.

TABLE 6: OCT in vitreoretinal interface syndrome.

(i) OCT is the most sensitive method to diagnose vitreomacular adhesions and the subsequent macular edema.

(ii) Two patterns of adhesions were identified: focal and multifocal. The focal one is associated with the macular hole and the multifocal one with the epimacular membranes.

(iii) The correlation between the visual acuity and the macular thickness measured by OCT is good in the preoperative period, but it does not exist postoperatively.

an incomplete posterior vitreous detachment and multifocal adhesions to the macula separated by areas of posterior vitreous detachment that appear as optically clear on OCT. Focal vitreomacular adhesions are associated with vitreoretinal traction syndrome and macular holes, whereas multifocal adhesions were identified in association with the epiretinal membranes. Epiretinal membranes contain collagen that appears on OCT as a relatively reflective thin band above the reflective retina, with or without optically clear spaces between them [6]. The epiretinal membrane has to be differentiated from the posterior hyaloid which is less reflective and thinner and from the highly reflective retinal fiber layer. The most common OCT finding in patients with epiretinal membranes is the thickening of the retina and the disappearance of the foveal pit. OCT is useful also for predicting the visual outcome after the epiretinal membrane removal. Preoperative cystoid macular edema seems to be the most important indicator of visual acuity following surgery. Preoperatively, there is a correlation between visual acuity and the macular thickness measured by OCT. The correlation is not valid postoperatively: the macular thickness tends to remain greater, although the visual acuity improves [6]. One possible explanation for this phenomenon is that the retinal gliosis prevents the macula from recovering its normal structure in the postoperative phase [6]. The role of OCT in vitreoretinal interface syndrome is summarized in Table 6 .

In Figures 6(a) and 6(b) is illustrated the role of OCT in identifying the relationship between the posterior vitreous and the retina. In this case, at the point of vitreomacular adhesion, there is a considerable thickening of the retina which is obvious both on the $3 \mathrm{D}$ picture of the macular cube and on the macular thickness map.

\section{Future Directions in the Development of OCT Technology}

The scope of OCT imaging in investigating the retinal diseases is continuously expanding. The limited axial and transverse resolutions are the main shortcomings of the current OCT technologies. The limitation of the axial resolution is determined by the so-called speckle noise: image scattering by the ocular structures. The lateral resolution is reduced by the restricted numerical aperture of the optical system [12]. The most significant future directions in OCT technology are swept source OCT, adaptive optics, and enhanced depth imaging.

4.1. Swept Source OCT (SS-OCT). In SS-OCT the light source has a wavelength of approximately $1,050 \mathrm{~nm}$. The superluminescent diode laser is replaced by a short cavity-swept laser. The frequencies of the emission can be rapidly tuned over a broad bandwidth $[26,28]$. Scan rates of 100,000-400,000 A-scan/second, with the axial resolution of $5.3 \mu \mathrm{m}$ over a 4-mm imaging range, are obtained by the use of a highspeed complementary metal oxide semiconductor camera (CMOS) and two parallel photodetectors [29]. If the system is coupled with adaptive optics, images to the level of individual photoreceptors are generated. The advantages of SS-OCT over SD-OCT are the following ones: the signals at the edges of the B-scan are reduced; the sensitivity improves with depth; the image range is longer: approximately $7.5 \mathrm{~mm}$. This last feature makes it possible to evaluate the anterior segment of the eye without using complex imaging techniques that might generate errors [7]. However, SS-OCT is limited by the fact that even with best patient cooperation, images are still subject to artifacts. Therefore, various algorithms had to be developed in order to improve the resolution [30].

4.2. Adaptive Optics. By correcting the ocular aberrations during the acquisition process, image resolutions at the cellular level became possible. The new algorithms limit the motion artifacts, increase the lateral resolution, reduce the speckle, and enhance the sensitivity. If the pupil is $>6 \mathrm{~mm}$, a lateral resolution of $2-3 \mathrm{~mm}$ is possible, which allows to identify the individual cones on a three-dimensional basis, the cross-sectional profiles of the nerve fiber bundles, and the foveal capillaries [30].

4.3. Enhanced Depth Imaging OCT. The potential application of OCT in the sub-RPE space and choroid is limited by its shallow penetration: approximately $1-3 \mathrm{~mm}$. The degree of choroidal penetration is determined by several factors: the proportion of scattered photons, the absorption spectrum of water, the scatter by the ocular media, the absorption by melanin [8]. Photon scattering is a phenomenon that influences the image formation in OCT: photons that are singly scattered add to the OCT signal, whereas photons that 


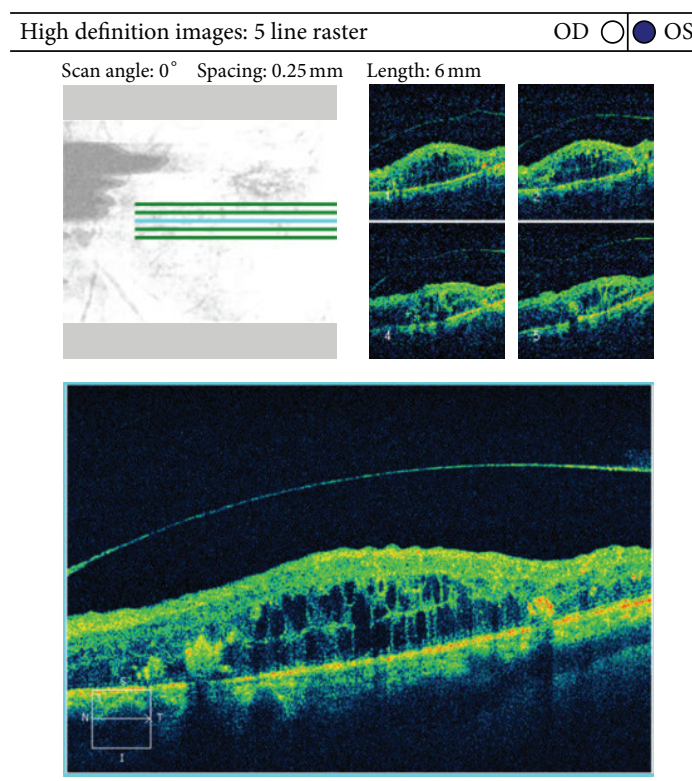

(a)
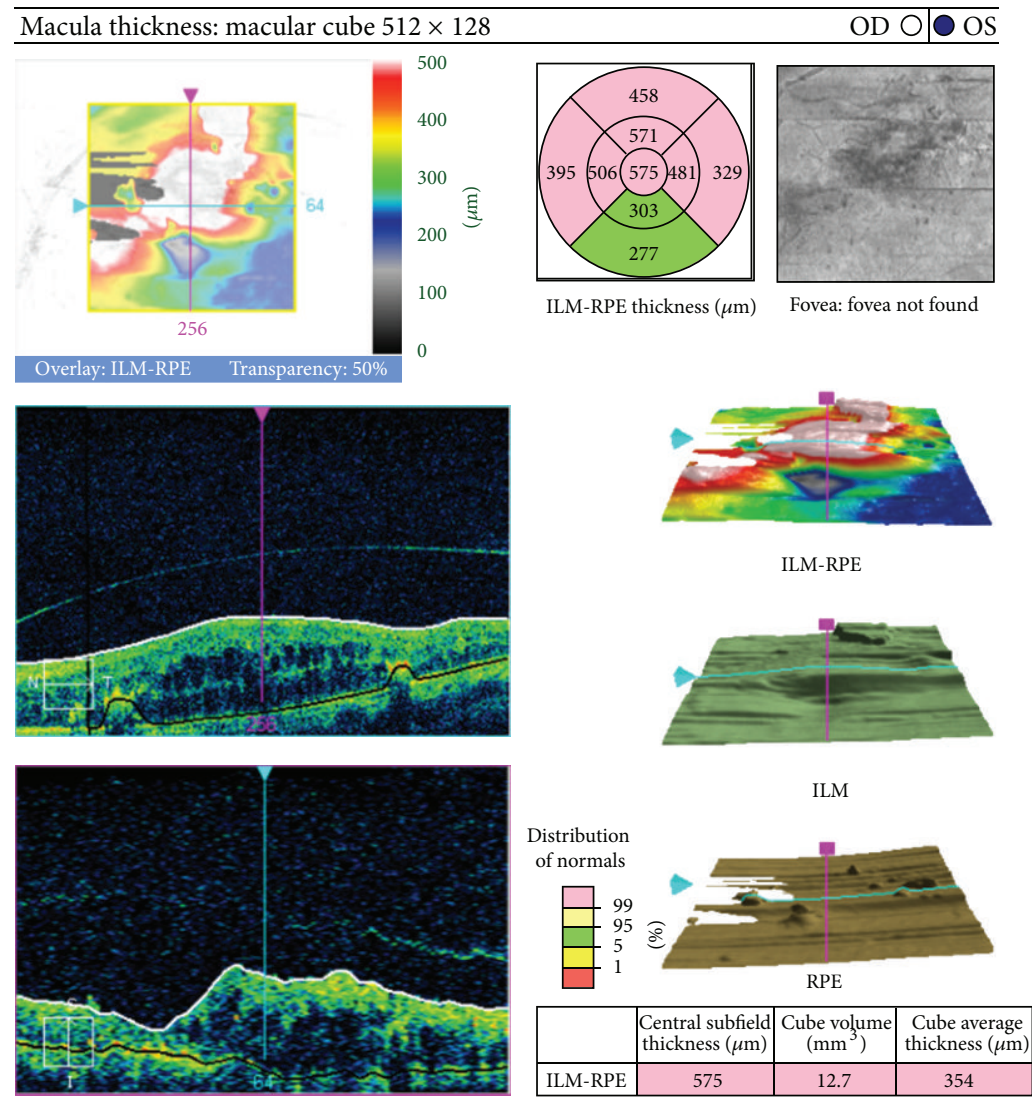

ILM-RPE
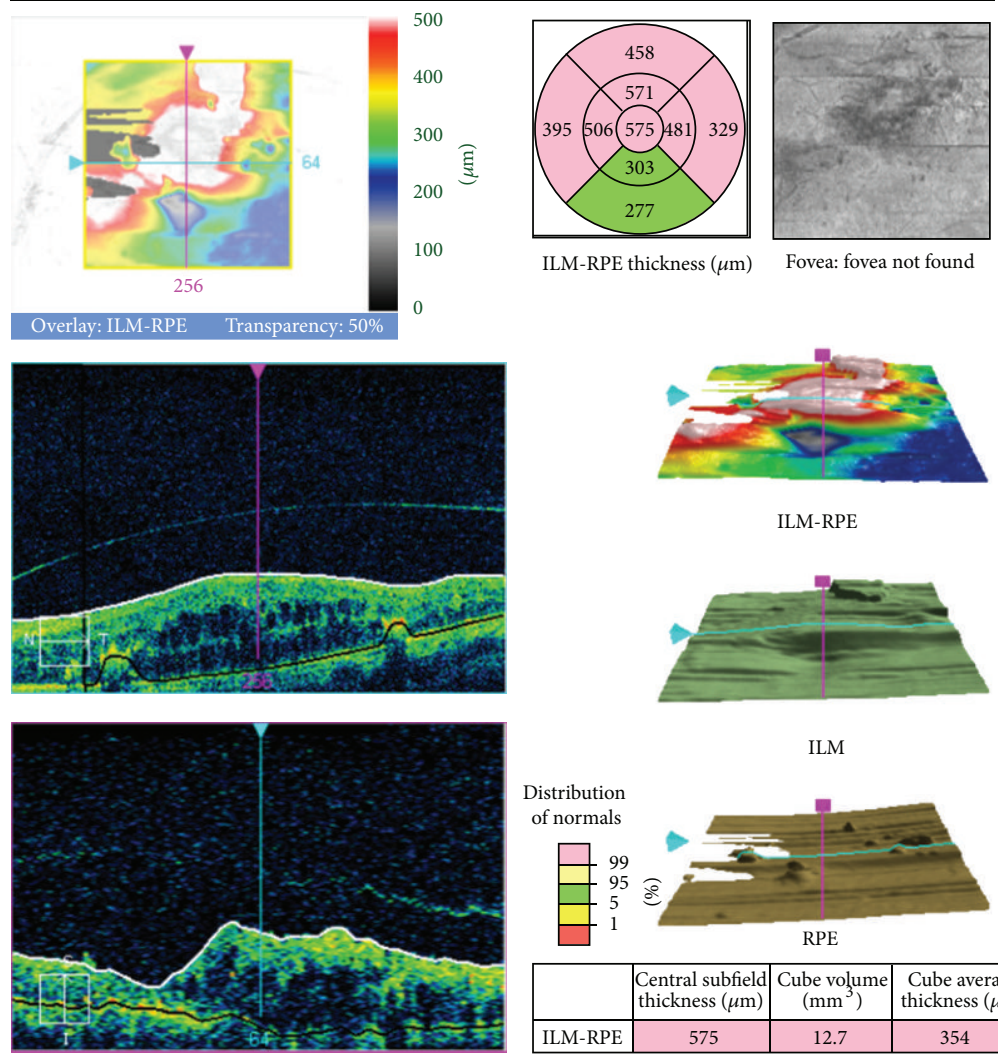

ILM-RPE thickness $(\mu \mathrm{m}) \quad$ Fovea: fovea not found

(b)

Figure 5: (a) and (b): Diabetic macular edema caused by vitreomacular traction.

are scattered multiple times contribute to the background noise [31]. The large amount of water within the eye limits the light wavelengths that can be used [32]. The absorption spectrum of water has two regions where the light absorption is low: at approximately $950 \mathrm{~nm}$ and between $1,000-1,100 \mathrm{~nm}$ [30]. The devices with wavelengths in the range of 1,000$1,100 \mathrm{~nm}$ can be used for the enhanced sub-RPE imaging, with ultrahigh-speed image acquisition and axial resolution in the 


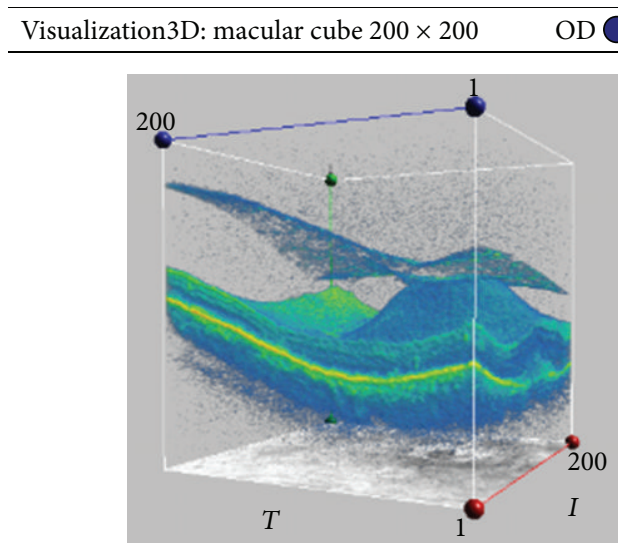

(a)
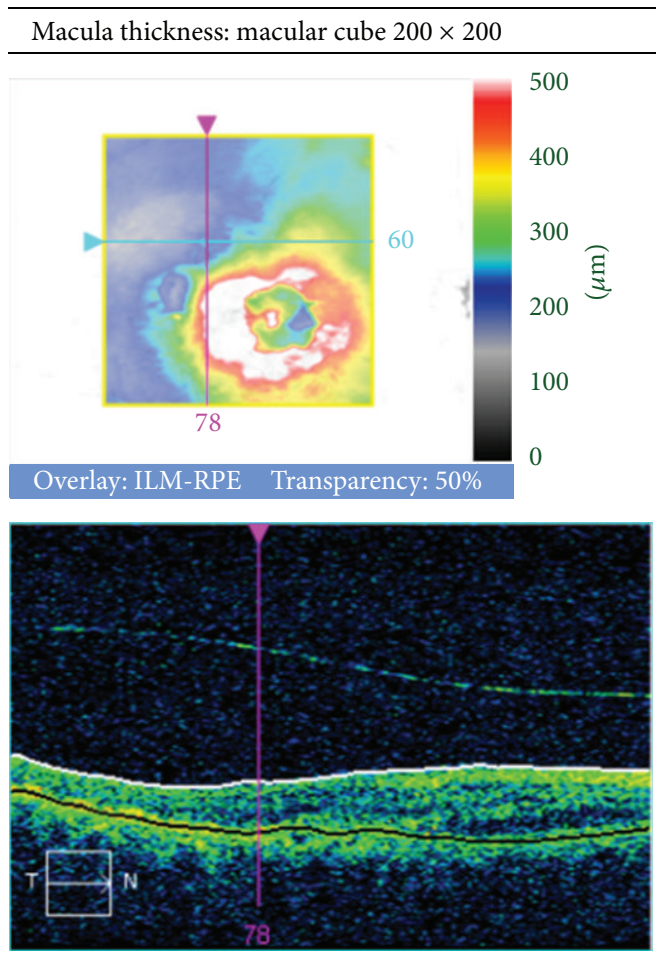

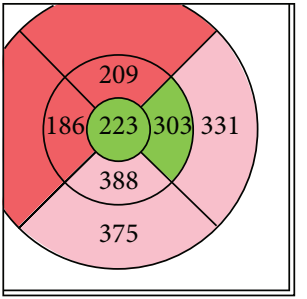

ILM-RPE thickness $(\mu \mathrm{m})$

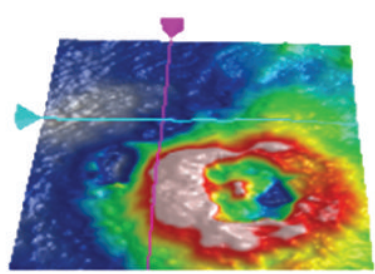

ILM-RPE

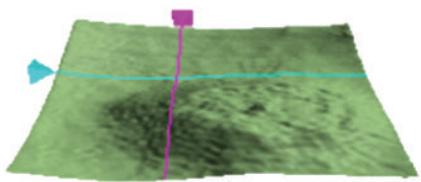

ILM

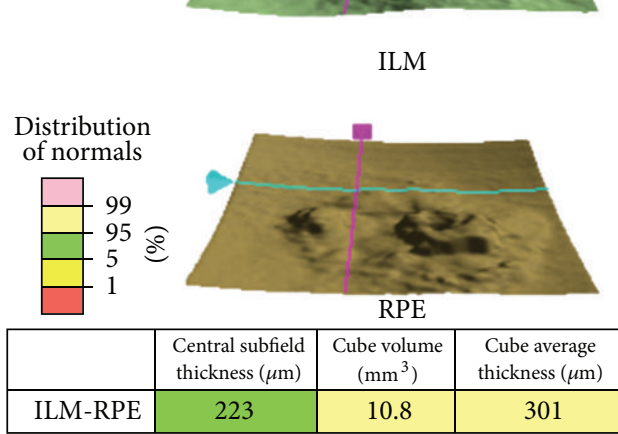

(b)

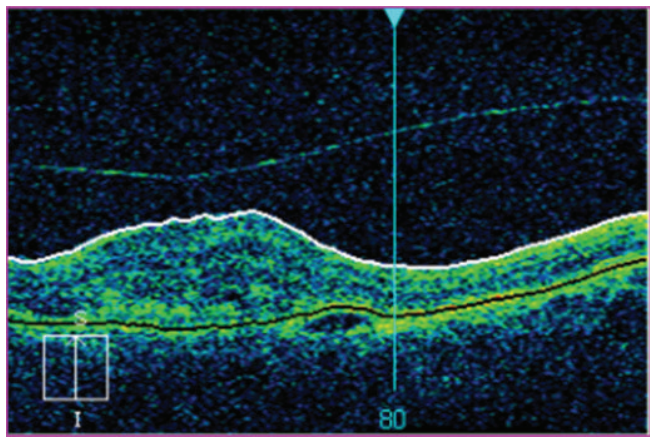

FIgURE 6: (a) and (b): Vitreomacular traction syndrome. 
range of $8 \mu \mathrm{m}$ [8]. This is useful in the managements of subRPE space diseases, particularly in AMD.

Enhanced depth imaging OCT (EDI-OCT) relies on placing the objective lens of the SD-OCT device closer to the eye, which allows the deeper structures to be localized closer to the zero delay. Subsequently, the choroid is better visualized. EDI-OCT is combined with other technological improvements: high-speed scanning, eye-tracking system, image-averaging technology, reduced noise, and greater coverage of the macula. As a consequence, the choroid is visualized as high resolution, repeatable, and reliable images [33].

\section{Discussion}

The invention of OCT in the 1990s represents a major progress in ocular imaging, as it is a fast, noninvasive, radiation-free examination technique, able to visualize the retinal layers at the microscopic level. The information offered by OCT is detailed but easily interpretable. OCT brought significant contributions in the field of diagnosis and monitoring a variety of retinal diseases, such as AMD, central serous chorioretinopathy, macular hole, vitreomacular interface syndrome, and diabetic maculopathy. OCT is applied by two main methods: time domain OCT (TD-OCT) and spectral domain OCT (SD-OCT). TD-OCT produces two-dimensional images of the sample internal structure. With SD-OCT the image is acquired rapidly-about 60 times faster than with TD-OCT. The image resolution in SDOCT is between $3-7 \mu \mathrm{m}$, as compared to $10-15 \mu \mathrm{m}$ in TDOCT. The practical impact of this improvement in resolution is the early detection of changes associated with retinal diseases, allowing a more rapid therapeutical intervention. The great number of scans done per second allows SD-OCT systems to generate $3 \mathrm{D}$ reconstructions, which can be further manipulated. Visualization of these data in 3D demonstrates subtle pathology that are not evident with conventional $2 \mathrm{D}$ images. The most frequent application of OCT in retinal disease is the measurement and monitoring of the macular thickness. The TD-OCT system (Stratus) gives a macular thickness map which is calculated from 6 radial B-scans crossing at the fovea. By interpolating data from these scans, the average macular thickness is calculated in 9 subfields centered on the fovea. Similarly, the total macular volume is obtained. SD-OCT provides images with much higher resolution. Given the differences between the measurements with the two types of OCT (TD and SD), algorithms are necessary in order to establish correlations between them. Ideally, the OCT scan must be interpreted in correlation with the fundus photography, direction of scan, and, ideally, the angiography. Despite the obvious advantages offered by OCT at the present moment, efforts are made for future developments and improvement.

\section{References}

[1] K. Khaderi, K. A. Ahmed, and G. L. Berry, "Retinal imaging modalities: advantages and limitations for clinical practice," Retinal Physician, vol. 8, no. 3, pp. 44-48, 2011.
[2] S. D. Talu and S. Talu, "Use of OCT imaging in the diagnosis and monitoring of age related macular degeneration," in Age Related Macular Degeneration. The Recent Advances in Basic Research and Clinical Care, G.-S. Ying, Ed., Intech, Rijeka, Croatia, 2012.

[3] D. Huang, E. A. Swanson, C. P. Lin et al., "Optical coherence tomography," Science, vol. 254, no. 5035, pp. 1178-1181, 1991.

[4] M. R. Hee, C. A. Puliafito, C. Wong et al., "Quantitative assessment of macular edema with optical coherence tomography," Archives of Ophthalmology, vol. 113, no. 8, pp. 1019-1029, 1995.

[5] N. G. Ghazi, T. Kirk, S. Allam, and G. Yan, "Quantification of error in optical coherence tomography central macular thickness measurement in wet age-related macular degeneration," American Journal of Ophthalmology, vol. 148, no. 1, pp. 90.e296.e2, 2009.

[6] F. G. Holz and R. F. Spaide, Essentials in Ophthalmology, Medical Retina, Berlin, Germany, 2005.

[7] R. A. Costa, M. Skaf, L. A. S. Melo et al., "Retinal assessment using optical coherence tomography," Progress in Retinal and Eye Research, vol. 25, no. 3, pp. 325-353, 2006.

[8] P. A. Keane, H. Ruiz-Garcia, and S. R. Sadda, "Clinical applications of long-wavelength $(1,000-\mathrm{nm})$ optical coherence tomography," Ophthalmic Surgery, Lasers and Imaging, vol. 42, pp. S67-S74, 2011.

[9] B. J. Lujan, "Revealing Henle’s fiber layer using spectral-domain OCT," Retinal Physician, vol. 8, no. 1, pp. 16-17, 2011.

[10] S. Talu, F. Balta, and S. D. Talu, "Fourier-domain optical coherence tomography in diagnosing and monitoring of retinal diseases," IFMBE Proceedings, vol. 26, no. 1, pp. 261-266, 2009.

[11] B. Lumbroso and M. Rippoli, Guide to Interpreting Spectral Domain Optical Coherence Tomography, James Allyn, Dublin, Calif, USA, 2010.

[12] M. Wojkowski, B. Kaluzny, and R. J. Zawadzi, "New directions in ophthalmic optical coherence tomography," Optometry and Vision Science, vol. 89, no. 5, pp. 524-542, 2012.

[13] D. F. Kiernan, W. F. Mieler, and S. M. Hariprasad, "Spectraldomain optical coherence tomography: a comparison of modern high-resolution retinal imaging systems," American Journal of Ophthalmology, vol. 149, no. 1, pp. 18.e2-31.e2, 2010.

[14] T. Murakami, K. Nishijima, T. Akagi et al., "Optical coherence tomographic reflectivity of photoreceptors beneath cystoid spaces in diabetic macular edema," Investigative Ophthalmology and Visual Science, vol. 53, no. 3, pp. 1506-1511, 2012.

[15] M. Menke, C. Lala, C. Framme, and S. Wolf, "The ever-evolving role of imaging in DME management," Retinal Physician, vol. 9, no. 4, pp. 24-32, 2012.

[16] D. F. Kiernan, S. M. Hariprasad, E. K. Chin, C. L. Kiernan, J. Rago, and W. F. Mieler, "Prospective comparison of cirrus and stratus optical coherence tomography for quantifying retinal thickness," American Journal of Ophthalmology, vol. 147, no. 2, pp. 267.e2-275.e2, 2009.

[17] F. Sulzbacher, C. Kiss, M. Munk et al., "Diagnostic evaluation of type 2 (classic) choroidal neovascularization: optical coherence tomography, indocyanine green angiography, and fluorescein angiography," American Journal of Ophthalmology, vol. 152, pp. 799-806, 2011.

[18] G. Coscas, Optical Coherence Tomography in Age-Related Macular Degeneration, Springer Medizin, Heidelberg, Germany, 2009.

[19] R. F. Spaide, "Enhanced depth imaging optical coherence tomography of retinal pigment epithelial detachment in agerelated macular degeneration," American Journal of Ophthalmology, vol. 147, no. 4, pp. 644-652, 2009. 
[20] F. M. Penha, P. J. Rosenfeld, G. Gregori et al., "Quantitative imaging of retinal pigment epithelial detachments using spectral-domain optical coherence tomography," American Journal of Ophthalmology, vol. 153, pp. 515-523, 2012.

[21] F. Mojana, L. Cheng, D. U. G. Bartsch et al., "The role of abnormal vitreomacular adhesion in age-related macular degeneration: spectral optical coherence tomography and surgical results," American Journal of Ophthalmology, vol. 146, no. 2, pp. 218.e1-227.e1, 2008.

[22] S. Jun Lee, C. S. Lee, and H. Jun Koh, "Posterior vitreomacular adhesion and risk of exudative age-related macular degeneration: paired eye study," American Journal of Ophthalmology, vol. 147, no. 4, pp. 621.e1-626.e1, 2009.

[23] I. Krebs, W. Brannath, C. Glittenberg, F. Zeiler, J. Sebag, and S. Binder, "Posterior vitreomacular adhesion: a potential risk factor for exudative age-related macular degeneration?" American Journal of Ophthalmology, vol. 144, no. 5, pp. 741.el746.el, 2007.

[24] V. Manjunath, J. Goren, F. J. Fujimoto et al., "Analysis of choroidal thickness in age-related macular degeneration using spectral-domain optical coherence tomography," American Journal of Ophthalmology, vol. 152, pp. 663-668, 2011.

[25] P. L. Penfold and J. M. Provis, Macular Degeneration, Springer, Berlin, Germany, 2005.

[26] P. A. Keane and S. R. Sadda, "Predicting visual outcomes for macular disease using optical coherence tomography," Saudi Journal of Ophthalmology, vol. 25, no. 2, pp. 145-158, 2011.

[27] A. C. Sull, L. N. Vuong, L. L. Price et al., "Comparison of spectral/Fourier domain optical coherence tomography instruments for assessment of normal macular thickness," Retina, vol. 30, no. 2, pp. 235-245, 2010.

[28] B. Potsaid, B. Baumann, D. Huang et al., "Ultrahigh speed $1050 \mathrm{~nm}$ swept source/Fourier domain OCT retinal and anterior segment imaging at 100,000 to 400,000 axial scans per second," Optics Express, vol. 18, no. 19, pp. 20029-20048, 2010.

[29] C. V. Regatieri, L. Branchini, and J. S. Duker, "The role of spectral-domain OCT in the diagnosis and management of neovascular age-related macular degeneration," Ophthalmic Surgery, Lasers and Imaging, vol. 42, pp. S56-S66, 2011.

[30] V. Raiji, A. Walsh, and S. Sadda, "Future directions in retinal optical coherence tomography," Retinal Physician, vol. 9, no. 4, pp. 33-37, 2012.

[31] U. Sharma, E. W. Chang, and S. H. Yun, "Long-wavelength optical coherence tomography at 1.7 microm for enhanced imaging depth," Optics Express, vol. 16, no. 24, pp. 19712-19723, 2008 .

[32] A. Unterhuber, B. Považay, B. Hermann, H. Sattmann, A. Chavez-Pirson, and W. Drexler, "In vivo retinal optical coherence tomography at $1040 \mathrm{~nm}$-enhanced penetration into the choroid," Optics Express, vol. 13, no. 9, pp. 3252-3258, 2005.

[33] R. F. Spaide, H. Koizumi, and M. C. Pozonni, "Enhanced depth imaging spectral-domain optical coherence tomography," American Journal of Ophthalmology, vol.146, no. 4, pp. 496-500, 2008. 

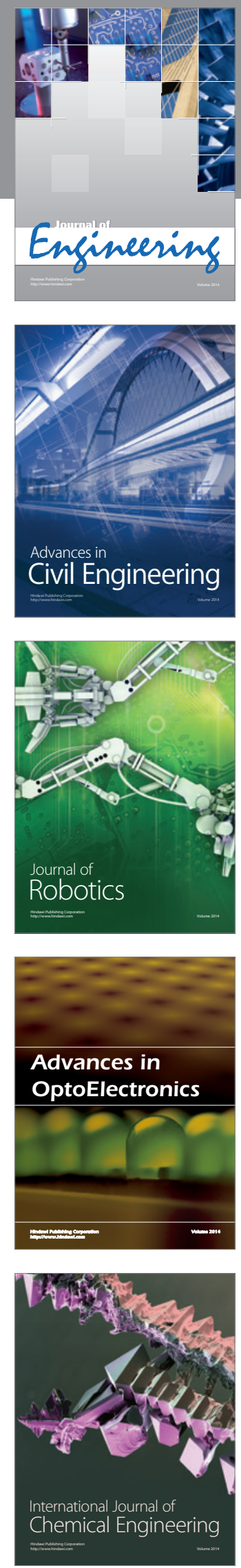

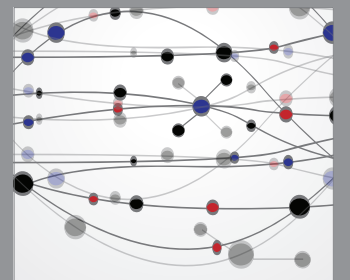

The Scientific World Journal
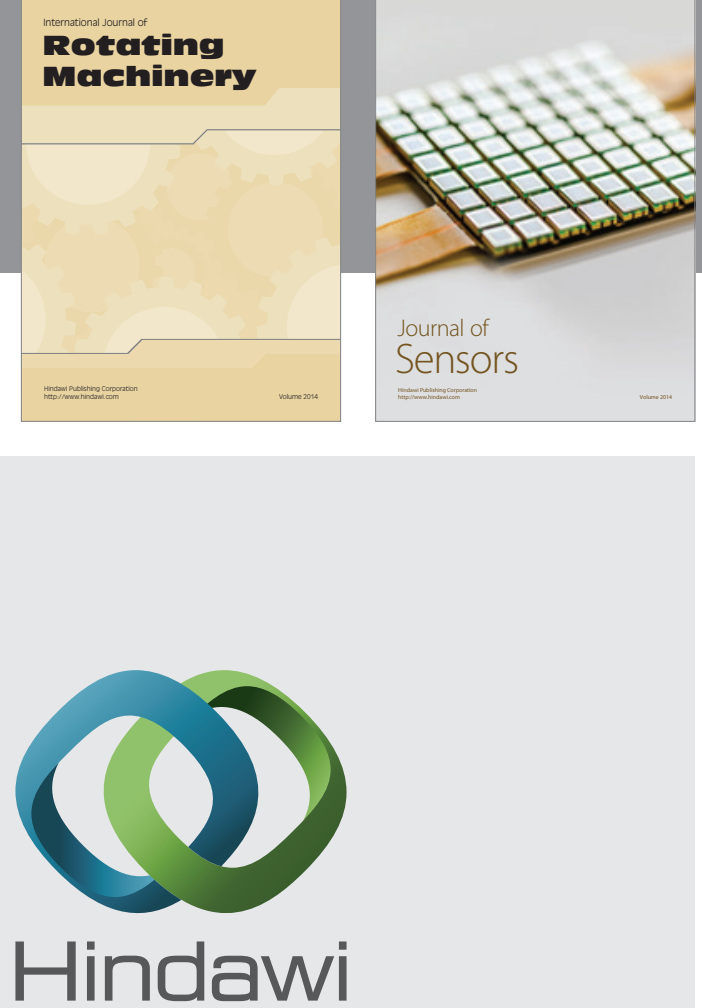

Submit your manuscripts at http://www.hindawi.com
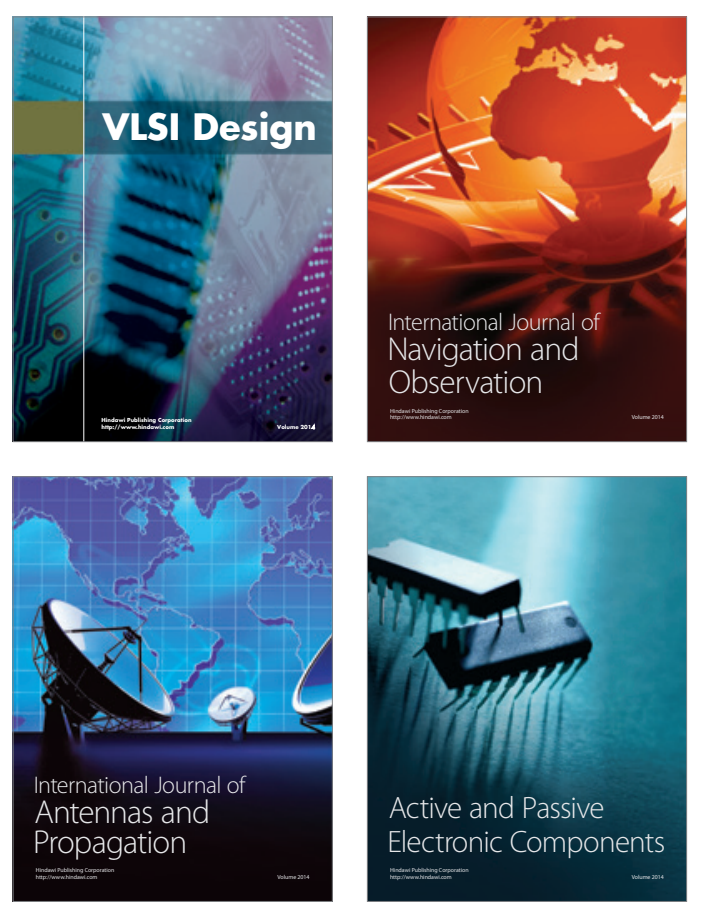
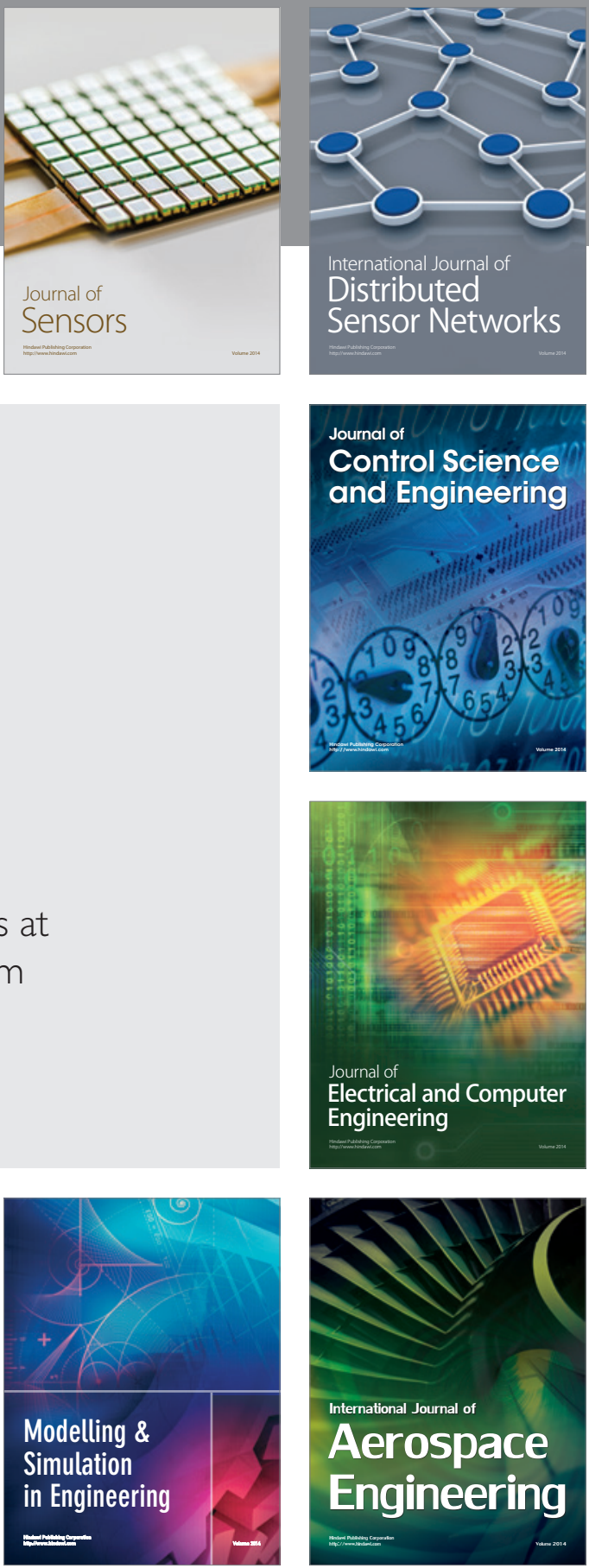

Journal of

Control Science

and Engineering
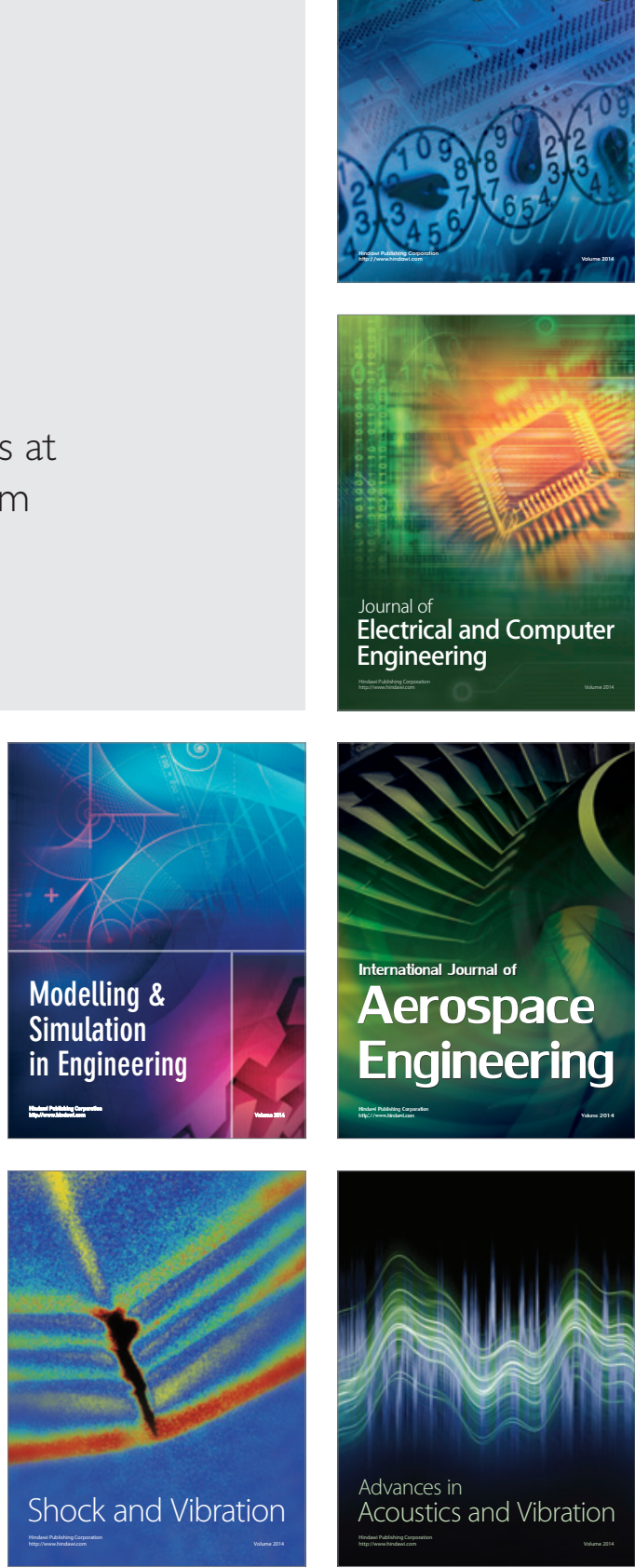